\title{
Prevalencia de bruxismo de vigilia evaluado por auto-reporte en relación con estrés, ansiedad y depresión
}

\author{
Association among stress, depression and anxiety with diurnal bruxism prevalence. \\ Miriam Patricia Ordóñez-Plaza ${ }^{1, a}$, Ébingen Villavicencio-Caparóo ${ }^{3, b}$, Omar Ricardo Alvarado-Jiménez ${ }^{2, c}$, María \\ Elizabeth Vanegas-Avecillas ${ }^{1, d}$.
}

\section{RESUMEN}

Objetivos: El objetivo de este estudio fue relacionar el estrés, la ansiedad y la depresión con la prevalencia del bruxismo probable de vigilia en los estudiantes universitarios de la Facultad de Odontología de la Universidad de Cuenca. Materiales y métodos: se realizó un diseño de casos y controles, donde se evaluaron en total 284 pacientes, se utilizó un cuestionario de auto reporte más la examinación clínica para determinar el bruxismo "probable" de vigilia y se aplicó la escala DASS-21 para medir el estrés, ansiedad, y depresión. Resultados: los datos revelaron una prevalencia de $52.1 \%$ de Bruxismo probable de vigilia y una asociación con las variables estrés (OR: 3.148 IC 95\%: 1.9 - 5.1), ansiedad (OR: 3.222 IC 95\%: 1.9 - 5.4) y depresión (OR: 3.284 IC 95\%: 2.0 - 5.3) con esta parafunción, a la vez no se encontró una asociación significativa entre bruxismo de vigilia con las variables edad mayor de 21 años y menor de 21 años (OR: 1.400 IC 95\%: O.8 - 2.2) y sexo (OR: 1.260 IC 95\%(0.7 - 2.0). Conclusiones: La depresión fue el factor asociado más relevante para el bruxismo de vigilia, esta patología es altamente prevalente entre los estudiantes universitarios de la facultad de odontología de la Universidad de Cuenca es de $52.1 \%$, existe una alta frecuencia de pacientes con estrés.

PALABRAS CLAVE: Bruxismo, factores asociados, auto-reporte, etiología, bruxismo de vigilia.

\section{SUMMARY}

Objectives: The objective of this study was to relate the stress, anxiety and depression with the prevalence of awake probable bruxism in college students of the Faculty of Dentistry at the University of Cuenca. Materials and methods: of cases and controls design, 284 patients were evaluated in total was conducted a questionnaire of self-report more clinical examination to determine the awake "probable" bruxism was used and the DASS-21

\footnotetext{
${ }^{1}$ Universidad de Cuenca. Cuenca, Ecuador.

2 Escuela de Odontología, Universidad de Cuenca, Ecuador.

${ }^{3}$ Universidad Católica de Cuenca. Cuenca, Ecuador.

a Especialista en Rehabilitación Oral.

b PhD. Cs. Salud Esp. En Bioestadística.

c Especialista en Rehabilitación Oral, Docente.

a Especialista en Rehabilitación Oral 
scale was applied to measure stress, anxiety, and depression. Results: The data revealed a prevalence of 52.1\% of awake probable bruxism and an association with stress variables (OR: 3.148, 95\% CI 1.9 - 5.1), anxiety (OR: $3.222,95 \%$ CI 1.9 - 5.4) and depression ( OR: 3.284; 95\% CI - 1.400 95\% CI: O 2.0 5.3) with this parafunction, while no less significant association between awake bruxism with the variables age over 21 and under 21 years (OR we not found 0.8 - 2.2) and sex (OR: 1.260 (95\% CI 0.7 - 2.0). Conclusions: depression was the most important factor associated to awake bruxism, this disease is highly prevalent among college students of the faculty of dentistry at the University of Cuenca is $52.1 \%$, there is a high frequency of patients with stress.

\section{KEYWORDS: Bruxism, associated factors, self-reported, etiology, awake bruxism.}

\section{INTRODUCIÓN}

El bruxismo es un hábito parafuncional caracterizado por apretamiento y frotamiento de los dientes durante el sueño o cuando se está despierto. Es considerado como una de las actividades parafuncionales más perjudiciales del sistema estomatognático, siendo considerado un factor de riesgo para trastornos temporomandibulares $y$, en particular para el dolor miofascial (1-3).

El bruxismo tiene dos manifestaciones circadianas distintas: El bruxismo nocturno o bruxismo del sueño (BS) que es una actividad inconsciente durante el sueño, asociada a micro-despertares, con manifestaciones musculares que inducen a rechinamiento dental(3-8). Y el Bruxismo diurno o bruxismo de vigilia $(B V)$ actividad semiconsciente que se produce al estar despierto, de la cual su manifestación preponderante es el apretamiento dentario(3-5.8).

Existen muchas técnicas disponibles para el diagnóstico del bruxismo. El uso de cuestionarios de Autoreporte sobre apretar o frotar los dientes durante el día se considera un parámetro de detección confiable para el bruxismo de vigilia según Fujisawa (9).

Cabe destacar que a pesar de una abundancia de técnicas, la confiabilidad y validez de herramientas de diagnóstico para el bruxismo de vigilia son escasas $(7,11,12)$. Por lo tanto el consenso internacional de expertos sobre bruxismo realizado en el año 2013 propone un enfoque reciente para la clasificación del bruxismo y, un sistema de clasificación diagnóstica de "posible", "probable", y "definitivo" del bruxismo de vigilia y del sueño con fines clínicos y de investigación (11).

Los integrantes de este consenso sugieren que el diagnóstico de Bruxismo "posible" debe basarse en el
Autoreporte por medio de cuestionarios o en la anamnesis como parte de una examinación clínica, el Bruxismo de Vigilia diagnosticado como "probable" se basa en el Autoreporte más la inspección positiva de signos/síntomas de bruxismo durante la examinación clínica (11); por lo menos 2 signos/síntomas de manifestaciones clínicas de bruxismo (13). Mientras que el diagnóstico de Bruxismo de Vigilia "definitivo" debe constar con el Autoreporte, examinación clínica positiva y electromiografía con grabaciones de los contactos dentarios (11).

El bruxismo es considerado de etiología multifactorial. Inicialmente se creía que éste tenía su origen en interferencias oclusales o por morfología dental alterada; sin embargo actualmente la evidencia de estudios genéticos indica que el bruxismo es causado por una combinación de factores genéticos y ambientales $(5,14,15)$.

El estrés, la ansiedad y la depresión son factores de riesgo muy comunes y que influencian estas vías en el BV, mientras sustancias psicoactivas y desordenes del sueño, tales como apnea del sueño, síndrome de movimiento de las extremidades y ronquidos, representan factores de riesgo comúnmente asociados con $\mathrm{BS}(1,6,14-18)$

El estrés, la ansiedad y depresión se miden por medio de la escala validada DASS-21(19).

La Prevalencia del bruxismo de vigilia a nivel mundial se encuentra entre $22.1 \%$ y $31 \%$; tanto a nivel nacional como local no existen datos estadísticos $(7,14,20)$. La mayor parte de evidencia científica se centra en el bruxismo del sueño, existiendo una gran falta de investigaciones del bruxismo de vigilia.

El objetivo de este estudio fue relacionar el estrés, la ansiedad y la depresión con la prevalencia del bruxis- 
mo probable de vigilia en los estudiantes universitarios de la Facultad de Odontología de la Universidad de Cuenca.

\section{MATERIAL Y MÉTODOS}

Diseño de tipo caso control. Se estudió una población finita, heterogénea, constituida por 461 estudiantes de 17 a 37 años de edad que estuvieron matriculados en la Facultad de Odontología de la Universidad de Cuenca durante el año 2015. El tamaño de la muestra calculado fue de 284 hombres y mujeres, considerando una prevalencia de $22.1 \%$ del bruxismo de vigilia, con un margen de error de 3\%. El muestreo fue aleatorio. Los criterios de inclusión fueron: todos los estudiantes de pregrado matriculados en la carrera de odontología durante el año 2015, y los criterios de exclusión fueron: estar embarazadas y no firmar el consentimiento informado. De los participantes incluidos se tomaron los casos y los controles, en caso de no aceptación, se escogió el siguiente participante de la aleatorización hasta completar el tamaño de muestra requerida.

Los datos fueron recolectados en un formulario diseñado previamente en el que constan los datos generales del paciente como edad, sexo, anamnesis, cuestionario de "autoreporte de bruxismo de vigilia".

\section{Autoreporte de Bruxismo de Vigilia $(13,14)$ :}

1. ¿Usted aprieta los dientes mientras está despierto?

2. ¿Usted frota los dientes mientras está despierto?

\section{Examen clínico para diagnosticar bruxismo de vi-} gilia. $(4,8,13)$.

1. Línea alba de la mucosa yugal.

2. Indentaciones linguales.

3. Hipertrofia de los músculos masticadores.

4. Sensibilidad dolorosa de los músculos masticadores.

5. Fracturas dentales y/o de restauraciones.

6. Movilidad dentaria sin presencia de enfermedad periodontal.

7. Presencia de facetas de desgaste dental producto del frotamiento o apretamiento dentario.

Para el diagnóstico de bruxismo la examinadora fue calibrada con docentes de la especialidad de Rehabilitación Oral de la Universidad de Cuenca, mientras que para el diagnóstico psicológico, fue calibrada por un especialista en psiquiatría del Hospital del Rio en Cuenca Ecuador.

Se dio un diagnóstico probable de bruxismo de vigilia a los estudiantes que indicaron autoreporte de bruxismo tal como recomienda Winocur (14) (la ratificación por parte del paciente a la pregunta 1 y/o 2 de dicho cuestionario de autoreporte), confirmado con la examinación clínica (si presenta 2 o más signos o síntomas de bruxismo según menciona Paesanni) (13).

Para evaluar la ansiedad, estrés y depresión a los estudiantes se les aplicó la escala DASS-21, que contiene 21 preguntas $(19,21)$ :

1. ¿Me costó mucho relajarme?

2. ¿Me di cuenta que tenía la boca seca?

3. ¿No podía sentir ningún sentimiento positivo?

4. ¿Se me hizo difícil respirar?

5. ¿Se me hizo dificil tomar la iniciativa para hacer cosas?

6. ¿Reaccioné exageradamente en ciertas situaciones?

7. ¿Sentí que mis manos temblaban?

8. ¿Sentí que tenía muchos nervios?

9. ¿Estaba preocupado por situaciones en las cuales podía tener pánico o en las que podría hacer el ridículo?

10. ¿Sentí que no tenía nada por qué vivir?

11. ¿Noté que me agitaba?

12. ¿Se me hizo difícil relajarme?

13. ¿Me sentí triste y deprimido?

14. ¿No toleré nada que no me permitiera continuar con lo que estaba haciendo?

15. ¿Sentí que estaba al punto de pánico?

16. ¿No me pude entusiasmar por nada?

17. ¿Sentí que valía muy poco como persona?

18. ¿Sentí que estaba muy irritable?

19. ¿Sentí los latidos de mi corazón a pesar de no haber hecho ningún esfuerzo físico?

20. ¿Tuve miedo sin razón?

21. ¿Sentí que la vida no tenía ningún sentido?

La prevalencia de bruxismo de vigilia se calculó por medio del análisis estadístico univariado. Para relacionar las variables se empleó el análisis estadístico bivariado Odss Rattio (OR), se tomó en cuenta un valor $\mathrm{p}<0.05$ y el intervalo de confianza del $95 \%$ como el nivel de significancia estadística. El software para la tabulación y análisis se desarrolló en el programa SPSS versión 21. 


\section{RESULTADOS}

La tabla 1 muestra que los dos grupos de pacientes estudiados son similares, sin presentar gran diferencia entre el sexo femenino y masculino con relación a la edad, lo cual indica que la muestra es simétrica tanto en la variable sexo como en la variable edad. El presente estudio muestra Figura 1 una población con la mitad de personas que presentan bruxismo probable de vigilia (apretamiento o frotamiento dental). En la Tabla 2 se aprecia que el grupo de personas con estrés tuvo más frecuencia de pacientes con bruxismo que el grupo que no tenía estrés. Los pacientes que presentaron estrés tuvieron 3 veces más probabilidad de desarrollar bruxismo que los pacientes sin estrés.
El grupo de personas que fueron diagnosticadas con presencia de ansiedad tuvieron más frecuencia de pacientes con bruxismo de vigilia que los que no la tenían Tabla 3. Los pacientes que presentaron ansiedad tienen 3 veces más probabilidad de desarrollar bruxismo que los pacientes sin ansiedad. Los pacientes que presentaron depresión tienen 3 veces más probabilidad de desarrollar bruxismo que los pacientes sin depresión (Tabla 4). Se identificó que el sexo no representa un factor de riesgo para tener bruxismo de vigilia, dado que el intervalo de confianza del estadístico OR incluye a la unidad (Tabla 5). Por otra parte la edad no representa un factor de riesgo para tener bruxismo de vigilia, dado que el intervalo de confianza del estadístico OR incluye a la unidad (Tabla 6).

Tabla 1.Distribución de la muestra de acuerdo a la edad y el sexo

\begin{tabular}{llcccccc}
\hline & & \multicolumn{2}{c}{ SEXO } & \multicolumn{3}{c}{ Total } \\
& & \multicolumn{2}{c}{ Femenino } & \multicolumn{2}{c}{ Masculino } & \multicolumn{3}{c}{} & $\%$ \\
\hline & $\mathrm{n}$ & $\%$ & $\mathrm{n}$ & $\%$ & $\mathrm{n}$ & 44.0 \\
$\begin{array}{c}\text { GRUPO DE } \\
\text { EDAD }\end{array}$ & 21 años o menos & 72 & 43.1 & 53 & 45.3 & 125 & \\
& Más de 21 años & 95 & 56.9 & 64 & 54.7 & 159 & 56.0 \\
& Total & 167 & 100 & 117 & 100 & 284 & 100 \\
\hline
\end{tabular}

Prevalencia de bruxismo probable de vigilia.

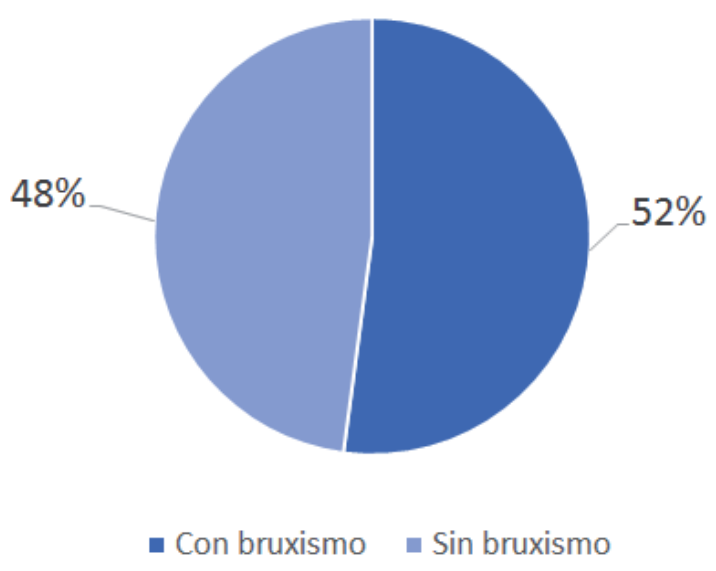

Figura 1. Prevalencia de bruxismo "probable" de vigilia 
Tabla 2. Asociación entre el estrés y el bruxismo de vigilia

\begin{tabular}{|c|c|c|c|c|c|c|c|}
\hline & & \multicolumn{4}{|c|}{ PRESENCIA DE ESTRÉS } & \multirow{2}{*}{\multicolumn{2}{|c|}{ Total }} \\
\hline & & \multicolumn{2}{|c|}{ Con } & \multicolumn{2}{|c|}{$\operatorname{Sin}$} & & \\
\hline & & $\mathrm{n}$ & $\%$ & $\mathrm{n}$ & $\%$ & $\mathrm{n}$ & $\%$ \\
\hline \multirow{2}{*}{$\begin{array}{c}\text { PRESENCIA DE } \\
\text { BRUXISMO }\end{array}$} & Con bruxismo & 80 & 68.4 & 68 & 40.7 & 148 & 52.1 \\
\hline & Sin Bruxismo & 37 & 31.6 & 99 & 59.3 & 136 & 47.9 \\
\hline \multicolumn{2}{|l|}{ Total } & 117 & 100 & 167 & 100 & 284 & 100 \\
\hline
\end{tabular}

OR: 3.148 IC 95\%(1.9-5.1)

Tabla 3. Relación entre la ansiedad y el bruxismo de vigilia.

\begin{tabular}{|c|c|c|c|c|c|c|c|}
\hline & & \multicolumn{4}{|c|}{ PRESENCIA DE ANSIEDAD } & \multicolumn{2}{|c|}{ Total } \\
\hline & & \multicolumn{2}{|c|}{ Con } & \multicolumn{2}{|c|}{$\operatorname{Sin}$} & \multirow[b]{2}{*}{$\mathrm{n}$} & \multirow[b]{2}{*}{$\%$} \\
\hline & & $\mathrm{n}$ & $\%$ & $\mathrm{n}$ & $\%$ & & \\
\hline \multirow{2}{*}{$\begin{array}{c}\text { PRESENCIA DE } \\
\text { BRUXISMO }\end{array}$} & Con bruxismo & 116 & 61.7 & 32 & 33.3 & 148 & 52.1 \\
\hline & Sin Bruxismo & 72 & 38.3 & 64 & 66.7 & 136 & 47.9 \\
\hline \multicolumn{2}{|c|}{ Total } & 188 & 100 & 96 & 100 & 284 & 100 \\
\hline
\end{tabular}

OR: 3.222 IC $95 \%(1.9-5.4)$

Tabla 4. Asociación entre la depresión y el bruxismo de vigilia

\begin{tabular}{cccccccc}
\hline & \multicolumn{2}{c}{ PRESENCIA DE DEPRESIÓN } & \multicolumn{2}{c}{ Total } \\
& & \multicolumn{2}{c}{ Con } & \multicolumn{2}{c}{ Sin } & & \\
& & $\mathrm{n}$ & $\%$ & $\mathrm{n}$ & $\%$ & $\mathrm{n}$ & $\%$ \\
\hline PRESENCIA DE & Con bruxismo & 105 & 64.4 & 43 & 35.5 & 148 & 52.1 \\
BRUXISMO & Sin Bruxismo & 58 & 35.6 & 78 & 64.5 & 136 & 47.9 \\
Total & & 163 & 100 & 121 & 100 & 284 & 100 \\
\hline
\end{tabular}

OR: 3.284 IC $95 \%(2.0-5.3)$ 
Tabla 5. Relación entre el sexo y el bruxismo de vigilia

\begin{tabular}{cccccccc}
\hline & \multicolumn{3}{c}{ SEXO } & \multicolumn{3}{c}{ Total } \\
& & \multicolumn{2}{c}{ Femenino } & \multicolumn{2}{c}{ Masculino } & \multicolumn{2}{c}{} \\
& & $\mathrm{n}$ & $\%$ & $\mathrm{n}$ & $\%$ & $\mathrm{~N}$ & $\%$ \\
\hline PRESENCIA DE & Con bruxismo & 91 & 54.5 & 57 & 48.7 & 148 & 52.1 \\
BRUXISMO & Sin Bruxismo & 76 & 45.5 & 60 & 51.3 & 136 & 47.9 \\
& & 167 & 100 & 117 & 100 & 284 & 100 \\
\hline
\end{tabular}

OR: 1.260 IC $95 \%(0.7-2.0)$

Tabla 6. Asociación entre la edad y el bruxismo de vigilia

\begin{tabular}{ccccccccc}
\hline & \multicolumn{4}{c}{ GRUPO DE EDAD } & \multicolumn{3}{c}{ Total } \\
& & 21 años o menos & Más de 21 años & & \\
& & $\mathrm{n}$ & $\%$ & $\mathrm{n}$ & $\%$ & $\mathrm{n}$ & $\%$ \\
\hline $\begin{array}{c}\text { PRESENCIA DE } \\
\text { BRUXISMO }\end{array}$ & Con bruxismo & 71 & 56.8 & 77 & 48.4 & 148 & 52.1 \\
& Sin Bruxismo & 54 & 43.2 & 82 & 51.6 & 136 & 47.9 \\
& Total & 125 & 100 & 159 & 100 & 284 & 100 \\
\hline
\end{tabular}

OR: 1.400 IC $95 \%(O .8-2.2)$

\section{DISCUSIÓN}

Recientemente algunas estrategias para el diagnóstico de bruxismo de vigilia se basan en registros electromiográficos ambulatorios, pero su costo y disponibilidad es limitado. Así una combinación de una entrevista (cuestionario) y una evaluación clínica realizada por personal calibrado, deben ser tomadas como el método más efectivo para establecer un diagnóstico de bruxismo de vigilia (1).

Los resultados en cuanto a la prevalencia de bruxismo de vigilia de la presente investigación Figura 1 coinciden con los presentados por Paesani et al., en donde el diagnóstico basado en entrevista más la examinación clínica fue de $52.5 \%$ (13). No obstante este estudio no coincide con datos de la revisión sistemática de Manfredini et al., que muestra los resultados de Jensen, el cual reporta una prevalencia de bruxismo de vigilia de $22.1 \%$ en sujetos que respondieron haber tenido esta parafunción a menudo, lo que se puede explicar debido a que el estudio de Jensen fue realizado solamente por medio de autoreporte, a diferencia del presente estudio que fue elaborado mediante autoreporte y examinación clínica (20). De igual forma en la misma revisión sistemática de Manfredini et al., 
que muestra los resultados de Winocur, el cual reporta $31 \%$ de prevalencia de bruxismo de vigilia en sujetos que la tuvieron durante los pasados seis meses, este estudio también fue hecho solamente con autoreporte (20). Por otra parte otra publicación que no concuerda con los resultados del presente estudio es el de Perlman et al., que revela una prevalencia de bruxismo de vigilia para los dos géneros de $19.2 \%$, esta discrepancia se explica debido a que su diagnóstico se realizó solamente por medio de autoreporte en cuestionarios (22). De igual forma hay discrepancia con el estudio de Sevgi Şener et al., que determinaron la prevalencia de bruxismo consciente (vigilia) en $33.9 \%$ en una población Turka, estudio que también se realizó mediante autoreporte (23). Los estudios de prevalencia que se realizan solamente mediante autoreporte subvalúan la magnitud de la patología entre desde 19 hasta $35 \%$.

Hasta la actualidad, existen criterios de diagnóstico validados solamente para el bruxismo del sueño y se basan en estudios polisomnográficos, los mismos que requieren el registro de la actividad de los músculos masticadores durante el sueño dentro de un laboratorio controlado $(13,20,24)$.

En base a la experiencia clínica, académica y evidencia científica se ha sugerido que el bruxismo de vigilia se asocia con una vida estresante y con ansiedad causada por responsabilidades familiares o presión laboral. Sin embargo la gran cantidad de evidencia científica sobre esta relación provienen de estudios que se centraron sobre el bruxismo del sueño; el presente estudio se realizó debido a la falta de investigaciones del bruxismo de vigilia $(2,15,25)$.

Existe una clara necesidad de estudios a gran escala sobre este tema y en particular la validación de herramientas de diagnóstico que permitan su uso en futuros estudios. En cuanto a la relación entre el estrés y el bruxismo de vigilia (Tabla 2) los resultados de la presente investigación son similares a los presentados por Perlman et al., en donde encontraron una correlación significativa entre bruxismo de vigilia con estrés $(\mathrm{p}=0.002)(22)$. Además autores como Winocur et al., demuestran resultados semejantes entre la asociación de autoreporte de bruxismo de vigilia con estrés emocional $(\mathrm{p}<0.05)(14)$. De igual forma Ahlberg et al., indican que las personas que autoreportaron bruxismo en su investigación mostraron mayor probabilidad de desarrollar esta parafunción al tener como factor de riesgo el estrés (OR: 2.5; IC 95\%: 1.5 - 4.2) (21). Al mismo tiempo Van Selms y Cols.(25) corroboran la asociación de bruxismo de vigilia (diagnosticado por medio de autoreporte) con estrés, su análisis estadístico indica que los pacientes tienen más probabilidad de desarrollar bruxismo de vigilia al tener estrés (OR $=1.36$ IC 95\%: 1.03-1.78).

Respecto a la relación entre ansiedad y bruxismo (Tabla 3) los resultados de la actual investigación concuerdan con los resultados presentados por Ahlberg et al., quienes indican que las personas con ansiedad tienen mayor probabilidad de desarrollar bruxismo de vigila, el mismo que se diagnosticó por medio de autoreporte (OR: 2.2; IC: 95\% 1.3 - 3.6) (21). A la vez coinciden del mismo modo Winocur et al., quienes revelaron la asociación significativa entre autoreporte de bruxismo de vigilia y ansiedad $(\mathrm{p}<0.001)(14)$. De igual forma Tavares et al., investigaron la relación entre los niveles de síntomas de ansiedad y la prevalencia de autoreporte de bruxismo de vigilia y, sus resultados demuestran una relación significativa $(\mathrm{OR}$ : 1.07) $(\mathrm{p}=0.05)$ entre ellos $(26)$.

Existe poca evidencia disponible de la asociación entre la depresión y el bruxismo de vigilia, sin embargo dicha asociación fue corroborada en los resultados del presente trabajo (Tabla 4) y a su vez son confirmados con los presentados por Van Selms et al., quienes evaluaron la asociación de bruxismo de vigilia (diagnosticado por medio de autoreporte) con depresión, el análisis de regresión múltiple indica que los pacientes tienen mayor probabilidad de desarrollar bruxismo de vigilia al tener depresión (OR $=1.4295 \%$ IC: $1.06-$ 1.89) (25).

Mientras que el sexo no se encuentra asociado con el bruxismo de vigilia, así lo demostraron los resultados de este estudio (Tabla 5) y, a su vez se encuentran en concordancia con los presentados por Winocur et al., y Perlman et al., en donde tener bruxismo de vigilia no se encuentra relacionado con el sexo de los pacientes $(14,22)$.

Por otro lado los resultados del estudio actual (Tabla 6) con respecto a la asociación entre la edad y el bruxismo de vigilia coincidieron con los reportados por Sevgi Şener et al., que en adultos jóvenes con un rango de edad entre 18 y 25 años, no encontraron 
asociación entre el bruxismo y la edad (23). A la vez las investigaciones de Winocur et al., y Manfredini et al., indican que no existen diferencias significativas entre la edad y la presencia de bruxismo de vigilia (14.20), incluso uno de ellos indica que el bruxismo disminuye con la edad (20). Por el contrario Perlman et al., encontraron una correlación estadísticamente significativa entre bruxismo de vigilia y edad $(\mathrm{p}=0.018)(22)$.

En conclusión, se observó que el bruxismo "probable" de vigilia es altamente prevalente entre los estudiantes universitarios de la facultad de odontología de la Universidad de Cuenca es de $52.1 \%$, existe una alta frecuencia de pacientes con estrés $(68.4 \%)$, los pacientes con bruxismo de vigilia tienen mayor predisposición de tener ansiedad (61.7\%), son vulnerables a este desorden psicosocial, se confirmó la asociación entre la depresión y el bruxismo probable de vigilia (64.4\%), de los pacientes que fueron diagnosticados con este tipo de bruxismo, no existe relación estadísticamente significativa entre el sexo y la edad con el bruxismo de vigilia.

\section{Correspondencia:}

\section{Miriam Patricia Ordóñez-Plaza \\ Correo electrónico: mpattyop@homail.com}

\section{REFERENCIAS BIBLIOGRÁFICAS}

1. Manfredini D, Lobbezoo F. Role of psychosocial factors in the etiology of bruxism. J Orofac Pain. 2009;23(2):153-66.

2. Bayar GR, Tutuncu R, Acikel C. Psychopathological profile of patients with different forms of bruxism. Clin Oral Investig. 2012; 16(1):305-11.

3. Santos-Miotto-Amorim C, Firsoff EFO, Vieira GF, Costa JR, Marques AP. Effectiveness of two physical therapy interventions, relative to dental treatment in individuals with bruxism: study protocol of a randomized clinical trial. Trials. 2014;15:8.

4. Paesani D. Bruxismo Teoria y Práctica. Chicago: Quintessence Publishing; 2012.

5. Castellanos J. Bruxismo: Nociones y conceptos. Rev ADM. 2015;72(2):63-9.

6. Quadri MFA, Mahnashi A, Al-Almutahhir A, et al. Association of awake bruxism with khat, coffee, tobacco, and stress among Jazan university students. Int J Dent. 2015;2015:1-5.

7. Hermesh H, Schapir L, Marom S, et al. Bruxism and oral parafunctional hyperactivity in social phobia ou- tpatients. J Oral Rehabil. 2015; 42(2):90-7.

8. Shetty S, Pitti V, Babu CLS, Kumar GPS, Deepthi BC. Bruxism: A literature review. J Indian Prosthodont Soc. 2010;10(3):141-8.

9. Fujisawa M, Kanemura K, Tanabe N, et al. Determination of daytime clenching events in subjects with and without self-reported clenching. J Oral Rehabil. 2013;40(10):731-6.

10. Koyano K, Tsukiyama Y, Ichiki R, Kuwata T. Assessment of bruxism in the clinic. J Oral Rehabil. 2008;35(7):495-508.

11. Lobbezoo F, Ahlberg J, Glaros AG, et al. Bruxism defined and graded: An international consensus. J Oral Rehabil. 2013;40(1):2-4.

12. Gómez S, Sánchez E, Castellanos J. Avances y limitaciones en el tratamiento del paciente con bruxismo. Rev ADM. 2015; 72(2):106-14.

13. Paesani DA, Lobbezoo F, Gelos C, Guarda-Nardini L, Ahlberg J, Manfredini D. Correlation between self-reported and clinically based diagnoses of bruxism in temporomandibular disorders patients. J Oral Rehabil. 2013;40(11):803-9.

14. Winocur E, Uziel N, Lisha T, Goldsmith C, Eli I. Self-reported Bruxism associations with perceived stress, motivation for control, dental anxiety and gagging. J Oral Rehabil. 2011;38(1):3-11.

15. Calic A, Peterlin B. Epigenetics and bruxism: Possible role of epigenetics in the etiology of bruxism. Int $\mathrm{J}$ Prosthodont. 2015;28(6):594-9.

16. Endo H, Kanemura K, Tanabe N, Takebe J. Clenching occurring during the day is influenced by psychological factors. J Prosthodont Res. 2011;55(3):159-64.

17. Hernández M. Estudio sobre el bruxismo y una nueva prueba de esfuerzo. Tesis doctoral. Murcia,España: Facultad de Medicina y Odontología, Universidad de Murcia; 2010.

18. Gungormus Z, Erciyas K. Evaluation of the relationship between anxiety and depression and bruxism. J Int Med Res. 2009;37(2):547-50.

19. Antúnez Z, Vinet E. Escalas de depresión, ansiedad y estrés (DASS-21): Validación de la versión abreviada en estudiantes universitarios chilenos. Ter psicológica. 2012;30:49-55.

20. Manfredini D, Winocur E, Guarda-Nardini L, Paesani D, Lobbezoo F. Epidemiology of bruxism in adults: a systematic review of the literature. J Orofac Pain. 2013;27(2):99-110.

21. Ahlberg J, Lobbezoo F, Ahlberg K, et al. Self-reported bruxism mirrors anxiety and stress in adults. Med Oral Patol Oral Cir Bucal. 2013;18(1):7-11.

22. Emodi Perlman A, Lobbezoo F, Zar A, Friedman Rubin P, van Selms MKA, Winocur E. Self-Reported bruxism and associated factors in Israeli adolescents. J Oral Rehabil. 2016;43:443-50.

23. Şener S, Karabekiroğlu S, Ünlü N. Genç yetişkin birey- 
lerde bruksizm farkındalığı ve ilişkili değişik faktörlerin değerlendirilmesi. Cumhur Dent J. 2014;17(4):361.

24. Lobbezoo F, Ahlberg J, Manfredini D, Winocur E. Are bruxism and the bite causally related? J Oral Rehabil. 2012;39(7):489-501.

25. Van-Selms MKA, Visscher CM, Naeije M, Lobbezoo F. Bruxism and associated factors among Dutch adolescents. Community Dent Oral Epidemiol. 2013;41(4):353-63.

26. Tavares LMF, da-Silva Parente Macedo LC, Duarte CMR, de Goffredo Filho GS, de Souza Tesch R. Cross-sectional study of anxiety symptoms and self-report of awake and sleep bruxism in female TMD patients. Cranio 2016;34(6):378-381.

Recibido : 18/03/2016

Aceptado: 12/05/2016 